\title{
Elder Abuse Screening Tools: A Systematic Review
}

\begin{tabular}{|r|l|}
\hline Journal: & The Journal of Adult Protection \\
\hline Manuscript ID & JAP-10-2016-0026.R1 \\
\hline Manuscript Type: & Research Paper \\
\hline Keywords: & Older people, old age, elder abuse, detection, screening, screening tools \\
\hline \multicolumn{2}{|}{} \\
\hline
\end{tabular}

SCHOLARONE ${ }^{\text {m }}$

Manuscripts 


\section{Introduction:}

Elder abuse is not a recent phenomenon but it has only been the subject of sustained interest and concern in recent years The UK charity Action on Elder Abuse has existed for many years and in 1995 developed a definition that is now used worldwide: 'Elder Abuse is a single or repeated act or lack of appropriate action, occurring within any relationship where there is an expectation of trust which causes harm or distress to the older person" (Action on Elder Abuse, 2017a).

Different words are used to describe abuse. These include: harm, exploitation, mistreatment and maltreatment. Elder abuse is broadly divided into the following categories: neglect, physical, psychological/emotional, financial and sexual. Most commonly found types are neglect and psychological or emotional, followed by physical abuse (Schofield and Mishra, 2003).There are longstanding difficulties in definitions and debates remain around definitions, even among experts (Pritchard, 1995). For example the issue of intimate partner violence/domestic abuse and elder abuse is complicated when it comes to a definition; Should those people who have been subject to intimate partner violence throughout life and who are now older be included as elder abuse victims? Or should the term only be used for individuals who experience abuse for the first time in later life? Neglect is also difficult to define: self-neglect, which refers to situations where an individual is unable to meet their own needs and often does not involve an external perpetrator, is a very different phenomenon to neglect by a caregiver, where chronic physical and mental health problems and a need for external assistance and support are usually key characteristics (National Research Council, 2003).

Over 500,000 people may risk experiencing abuse in the United Kingdom every year (Action on Elder Abuse, 2017b). Worldwide prevalence rates range from $1 \%$ to $35 \%$, depending on reporting methods and definitions used, but these figures are considered to be the tip of the iceberg due to underreporting(WHO, 2008).Elder abuse is difficult to detect. People may be embarrassed or afraid to report it. Many older people may make attempts to hide it rather than disclose situations and there are a range of reasons why someone might not wish to report abusive situations (Penhale, 1993). Incidents that may appear relatively minor can have a debilitating and long-lasting effect on older people (National Research Council, 2003) It may not be possible for an older person to recover and 'move-on' from the situation in the way that a younger person might; in addition the rates of morbidity and mortality following experiences of abuse are high for older people and the possible co-existence of co-morbid health conditions is also likely to contribute to this (Lachs et al, 1998)

Screening and the use of screening tools to assist in case finding may help in detection of abuse and neglect, but this needs to be handled sensitively by the professional using the tool. Screening should be situated within an overall system of case detection and management to ensure that any consequences of detection are dealt with in a considered and productive way for the victim and, as far as possible, would not lead to detrimental effects for the individual. Professionals must not only identify abuse but should also be able to provide further screening, follow up or referrals to other agencies as well as intervention and support (Raffle and Gray 2007).

The concept of screening is to accurately identify people who may be at risk of a disease/condition. Based in epidemiology and epidemiological principles the objective is to establish the presence (or not) of a risk factor, rather than to make a definitive diagnosis. The point is to highlight those cases that have a higher statistical probability of illness or disease and then follow those cases up by either further testing or treatment (Phelan and Treacy, 2011). Screening tools should be able to correctly detect those with disease or condition (in this case abuse or neglect) and those without. The idea is to raise the 'index of suspicion' of the professional undertaking the screening about the possibility of abuse and then to follow this up. Tools are evaluated by using statistical tests such as sensitivity (to correctly identify those who are being abused-true positives) and specificity (to correctly identify those not being abused-true negatives). A false positive occurs when a person is identified as being abused when they are not, whilst a false negative results in identifying those without the disease (or condition) when they actually do have this. The perfect test would be both highly sensitive and specific but in reality most screening tests cannot achieve both, with increases and decreases in sensitivity and specificity usually inversely related (Grimes and Schulz, 2002).

Elder abuse is complex, rarely occurs in isolation and ambiguities arise on closer examination, resulting in limitations when it comes to categorization (Darzins et al, 2009). Screening in elder abuse 
is multifaceted. It is not realistic to simply categorize people as abused or as not abused. In clinical practice, human beings do not fit neatly into a sensitivity and specificity effectiveness concept (Grimes and Schulz, 2002). There are some clear ethical risks to screening such as, the consequences of false positives (incorrect accusations of abuse) or false negatives (missing cases of abuse where an intervention may save lives). Such incidents could have devastating and far reaching effects for the individuals involved. Unlike screening in physical health, such as cervical screening in order to detect potential cervical cancer, there are more nuanced situations specific to elder abuse that requires careful and ethical consideration. For example elder abuse victims may not have requested or wanted investigation (Raffle and Gray, 2007). Also a number of health problems and their complexities in older age may mimic and overlap symptoms of abuse resulting in a reluctance among professionals to become involved as they may fear potential errors of judgement in determining whether abuse may have occurred or not, or that an intervention might do more harm than good (Cohen 2011).

A number of well-known screening tools exist. The Brief Abuse Screen for the Elderly (BASE) and the Indicators of Abuse (IOA) screening tools are among the earliest to have been developed (Reis and Nahmiash, 1998). Fulmer et al (2005) developed the Elder Assessment Instrument (EAI), revised in 2012, and this gives an overall assessment of an older person, including physical examination (Fulmer et al, 2005; Fulmer et al, 2012). The Elder Abuse Suspicion Index (EASI), developed in Canada, uses a combination of self-report items that the older person is asked, with an item for the assessing professional. This encourages reflection on the part of the professional in relation to an observation of the person for signs of abuse (Yaffe et al, 2008; Yaffe et al, 2012).

\section{Rationale for the review:}

There are persuasive reasons to screen for elder abuse. Evidence shows abuse is linked to a range of adverse health outcomes with higher prevalence of depression and dementia among those with substantiated elder abuse (Bitondo Dyer et al, 2000). As previously indicated there are increased mortality rates for older people who experience abuse. Health and Social Care environments may be the only contexts where older people are seen and have contact with others. This can be a valuable opportunity to detect abuse and a chance for victims to disclose and be offered support and assistance. If detected early enough, elder abuse victims can be offered the opportunity for an intervention, and help to reduce the risks they may be exposed to. This can prevent serious harm from occurring or even save lives.

With these points in mind, a decision was made to focus on the use of screening tools in elder abuse as the topic of an MSc in Clinical Research. The research component of this programme was to undertake a systematic review in a topic of interest to the student, in this instance elder abuse. The review was conducted in 2014-15.

\section{Aims of the review:}

1. Identify screening tools used to detect elder abuse in the health and social care environment.

2. Identify issues related to their use and effectiveness.

\section{Methods:}

One of the first steps in the review process is to determine the eligibility criteria on which the literature search would be based. Studies were selected with the Action on Elder abuse definition used as a guide to frame the decisions about the criteria to be used in the review; this includes the 'relationship of trust' statement in the definition. . The population that was the focus of the review was older people, living in their own homes with a significant other person or a caregiver. The emphasis was the relationship between the victim and the perpetrator, resulting in the exclusion of studies conducted in hospital or care home settings, as well as studies where the screening tool was tested in self-neglect. Although this latter type of abuse is very serious, it's characteristics are different and it does not involve an external perpetrator or the relationship of trust, which was the focus of this review. The principal focus in this study was thus older people living in the community in their own homes or with others such as relatives.

Included: Studies related to screening of:

- Population aged 55 and over 
- Older people living in own home (or with relatives, community dwelling)

- Literature published in English, between the years 1994-2014

- A tool tested with or by the victim

Excluded: Studies relating to screening of:

- Older people diagnosed with dementia

- Residential or care home settings

- Self-neglect

It is important to note here that elder abuse in these latter populations and settings is subject to different influences and characteristics, warranting its own separate investigation in terms of potential screening and case finding.

Information sources: The health and social care related databases AMED, CINAHL, MEDLINE, The Cochrane Library, SCOPUS, The King's Fund and NICE were used during the search process.

Search terms: Key words, such as, "elder", "old" "abuse", mistreatment", "questionnaire, "instrument" were combined using Boolean logic and then used to search the databases over a two-day period in November 2014. The search strategy began with identification of all possible eligible citations. Initial searches resulted in a large number of titles and abstracts, which were subsequently excluded as it was evident that they did not investigate elder abuse screening. Records that did include the keywords were examined in more detail, with a total of 188 records retrieved. On reviewing the abstracts of these records a further number of studies were excluded.

Additional search and selection strategies: The citations of the reviewed studies were searched which resulted in the inclusion of a further 5 eligible studies. The lead author and a second assessor $\left(2^{\text {nd }}\right.$ author) reviewed all papers and made decisions about the inclusion and exclusion of the studies for the full review. This process of consultation with co-authors continued throughout the entire systematic review process.

Preferred reporting items for systematic reviews and meta-analysis (PRISMA) guidance was followed (Moher et al, 2009). Figure 1 shows the study selection process.

Figure 1: PRISMA flowchart:

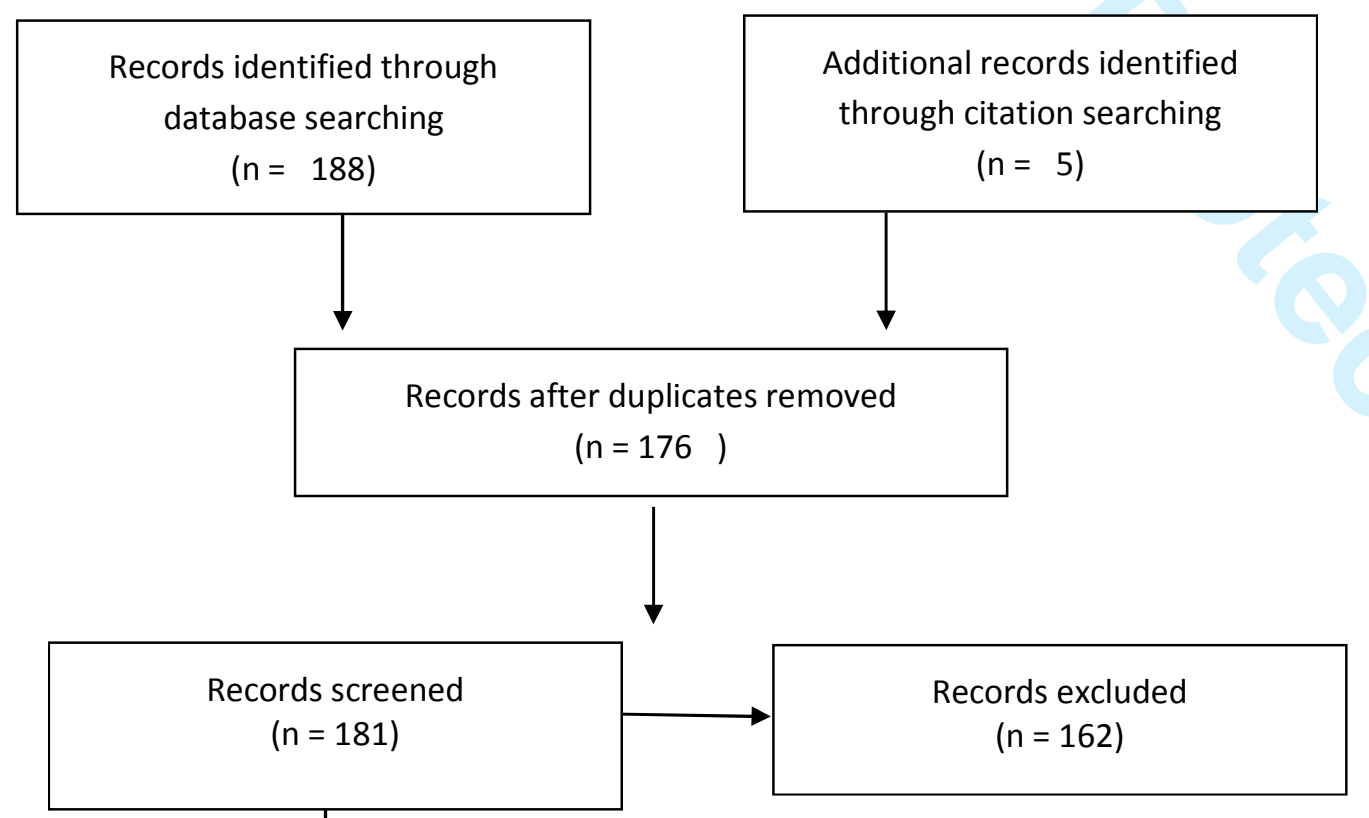


Table 1 Characteristics of tools

Data items and collection process: The items of data collected were: total number of participants recruited to study; number of questions in the tool; method of screening, i.e. face to face, selfcompletion; who completed the tool i.e. the clinician or the participant; clinical setting and country and statistical analysis used. A data collection form was adapted for use specifically to assist the systematic review process.

Analysis: A narrative synthesis approach was used for the analysis (Popay et al, 2006). Due to the heterogeneity between studies it was not possible to undertake a meta-analysis. Narrative synthesis refers to a method of summarizing and explaining data where it is not possible to use meta-analysis and where data from different study designs cannot be combined. The results are structured by the central conclusions of the individual studies and the characteristics of the tools which were related Ryan, 2013).

Results:

Synthesis of results:

Eleven studies met the study criteria (primary research in the use of screening tools) and were included in the review and analysis. Twelve screening tools were identified. Of these twelve, three were revised versions of tools (Expanded Indicators of Abuse (E-IOA), Elder Assessment InstrumentRevised (EAI-R) and the Elder Abuse Suspicion Index-self-administer (EASIsa). The tools varied in length and the types of abuse detected. The clinical settings where they were tested varied, as well variation in the methods to evaluate effectiveness. Only three tools (Expanded Indicators of Abuse, Elder Assessment Instrument and the Elder abuse Suspicion Index) were subjected to the more rigorous method of evaluation of sensitivity and specificity testing. Otherwise validity and reliability analysis was performed. Characteristics and descriptions of each screening tool are shown in Table one and in Table two.

\begin{tabular}{l} 
Name of tool \\
\hline Brief Abuse Screen for \\
the Elderly (BASE) (Reis \\
and Nahmiash, 1998) \\
\\
Indicators of Abuse \\
Screen (IOA) (Reis and \\
Nahmiash, 1998)
\end{tabular}

Description

Brief five item tool intended to determine urgency for making an onward referral. Takes approximately one minute to complete, is useful to determine probability of abuse. Recommended to be used with the Indicators of Abuse screen (see below).

Twenty seven items examining care giver and care receiver characteristics concurrently. It is considered easy to use as items reflect routine clinical examination. Completed as part of a clinical assessment resulting in potential completer subjectivity. Addresses risks 
factors not actual signs and symptoms (Reis and Nahmiash, 1998).

Expanded IOA (Cohen, 2013)

Elder Assessment Instrument(EAI) (Fulmer et al, 2005)

Elder Assessment Instrument-Revised (Fulmer et al, 2012)

Elder Abuse Suspicion Index (EASI) (Yaffe et al, 2008) and the EASI selfadminister (Yaffe et al, 2012)

Hwalek-Sengstock Elder Abuse Screen Tool (HSEAST) (Moody et al, 2000)

Vulnerability to Abuse Screening Scale (VASS) (Schofield and Mishra, 2003)

lowa Elder Abuse Screen (IEAS) (Buri et al, 2009)

Older Adult Financial Exploitation Measure (OAFEM) (Conrad et al, 2010a)

Older Adult Psychological Abuse Measure (OAPAM) (Conrad et al, 2010b)
A version of the Indicators of Abuse screen that was expanded to rectify the subjectivity identified in the original. Contains twenty one items and should be conducted as a semi structured interview.

Forty one items which assess signs and symptoms, subjective views, neglect, exploitation and abandonment. It does not use a scoring system, which may result in completer subjectivity. Psychological abuse is not assessed. It is lengthy to complete but does involve a physical examination of the person which allows observation of any evidence i.e. bruising.

Fifty one item adaptation of the above with items added to capture psychological and financial abuse. It is designed as an initial screen to highlight any need for more skilled assessment.

Six items in questions asked directly to the victim (covering all aspects of abuse), with one observational question for the person completing. There are five in the self-complete version (observational question not included). Easy to use and shown to increase awareness among clinicians. The self-complete version may allow fuller disclosure but at the cost of the observational item.

Contains fifteen items but a shortened nine item version exists. Summative scoring with a cut off of three or higher suggesting that more thorough assessment is needed.

Considered best used as part of a clinical interview with expert supervision.

Twelve items (ten from the Hwalek-Sengstock Elder Abuse Screen) in self-report format addressing the concepts of vulnerability, dependence, dejection and coercion. It does not measure neglect which, as one of the most common types of abuse, results in a significant drawback of the tool.

A combination of three tools (Hwalek-Sengstock Elder Abuse Screen Tool, Vulnerability to Abuse Screening Scale and a nine item screen for various types of abuse from the American Medical Association) resulting in a lengthy tool of thirty six questions and some repetition of items.

The only tool identified that assesses financial abuse. Initially it had seventy nine items but has now been refined to twenty five. It detects financial abuse that occurs outside the "trusting" relationship i.e. scams. It has been further developed in Ireland (Phelan et al, 2014).

Started as thirty two items, but has been refined to an eighteen item self-report tool. Addresses psychological abuse and is considered useful in determining severity of such abuse. It asks questions of both victim and carer, which is valuable as the characteristics of the carer are also significant in the detection of abuse (Phelan and Treacy, 2011).

Table 2: Review results:

\begin{tabular}{llllll}
\hline $\begin{array}{l}\text { Authors } \\
\text { (Year) }\end{array}$ & $\begin{array}{l}\text { Study } \\
\text { design }\end{array}$ & $\begin{array}{l}\text { Name of } \\
\text { Tool }\end{array}$ & $\begin{array}{l}\text { Number of } \\
\text { participants }\end{array}$ & $\begin{array}{l}\text { Clinical } \\
\text { setting/country }\end{array}$ & Study overview and findings \\
\hline $\begin{array}{l}\text { Cohen } \\
(2013)\end{array}$ & Validation & E-IOA & 1317 & $\begin{array}{l}\text { Emergency } \\
\text { rooms, social } \\
\text { welfare agencies, }\end{array}$ & $\begin{array}{l}\text { The original study it was conducted in } \\
\text { was not available for review in } \\
\text { English. The results in this study }\end{array}$
\end{tabular}




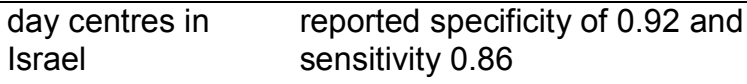

reported specificity of 0.92 and sensitivity 0.86

Doctors' waiting rooms in Montreal, Canada

Medical and New York

Adult Protection
Services in
Chicago

Adult Protection Services in Chicago

Social services Agency in lowa, USA

Family medical centres and community health and social centres in Montreal, Canada

\section{Emergency departments in Florida and $\mathrm{New}$ York}

Postal survey, part of a longitudinal study on women's health in Australia

Housing authority residents in Florida

Health and social service agency in one city in North dental clinics in
Tested the viability of the self-report version of the Elder Abuse Suspicion Index It was four times more likely to identify abuse when matched to use of the EASI tool

The only study which compared one tool with another and to the Conflict Tactics Scale, considered a gold standard in domestic abuse detection. Elder Assessment Instrument-Revised performed poorly when compared to the Conflict Tactics Scale

Part of a larger study (Older Adult Mistreatment Assessment) and an important first investigation into financial abuse as a separate type

Tested the psychometric properties of a tool to detect psychological abuse only, in the same population directly above. Requires further refinement

Tool was not predictive of abuse. The study found cognitive impairment to be an indicator of lower abuse scores

Specificity 0.75 , sensitivity 0.47 . The study compared the EASI with expert Social Worker Evaluation

Tool was used by researchers and compared to experts in the field. The researchers underreported abuse (5\%) compared to the experts who found it present in $22 \%$ of cases

Tested in a large sample. However as it was a postal survey it was not possible to substantiate any cases of abuse. Requires further testing

The study found that false negatives were more likely than false positives

High predictive validity $89-91 \%$ (how well a test predicts future behaviour) (Phelan and Treacy, 2011). True 
Narrative synthesis:

Six distinct types of screening tools were identified, grouped together according to tool similarities and purpose. The main findings are outlined below.

1. The Brief Abuse Screen for the Elderly with high predictive validity is a useful initial screen only when used with the Indicators of Abuse (which had high internal consistency). This can make it a lengthy process (2-3 hours) as it is used as part of an overall clinical assessment (Phelan and Treacy, 2011). This suggests that these tools have better utility in a research setting rather than health and social care environments. The expanded version of the IOA displayed the highest sensitivity of all the tools (0.92) but at the cost of poorer specificity of 0.86 . It has been further developed into the Mistreatment of Older Adults Risk Factors (MOARF)(Lindenbach et al, 2012)

2. The Elder Assessment Instrument and the Elder Assessment Instrument-Revised: The EAI displayed the highest specificity of all the tools $(0.93)$ but a weaker specificity of 0.71 . It does require further empirical testing (Phelan and Treacy, 2011). While the revised version is lengthy at 51 items, it can be administered in a 15 minute interview improving its utility for use in the busy health and social care environment.

3. The Elder Abuse Suspicion Index: The disappointing sensitivity (0.47) and specificity of 0.75 were subject to further analysis in the Yaffe et al 2008 study, when the overall sensitivity reduced to 0.32 when the first question, designed to address risk, was removed. This results in the interesting concept that the first question in a screen may in fact act as a primer for remaining items and allow fuller victim disclosure (Yaffe et al, 2008). This concept warrants further exploration.

4. The Hwalek-Sengstock Elder Abuse Screen showed good test-retest reliability (administering the test more than once) but poor internal reliability $(0.46)$ meaning that there is poor consistency of the items within the screen (Moody et al, 2000). The expanded version used in the Vulnerability to Abuse Screening Scale did show some promise with improved internal reliability (Cronbach's alpha 0.74). Disappointingly, when combined with the algorithm used by the American Medical Association in the lowa Elder Abuse Screen, it displayed poor ability to discriminate abuse from non-abuse.

5. The Older Adult Financial Exploitation Measure: With growing concerns about the extent of financial abuse, the development of this tool is welcomed but it does require shortening in length (25 items) and validation testing (Phelan and Treacy, 2014).

6. The Older Adult Psychological Abuse Measure: A good screening tool specifically for psychological abuse. Only experts in elder abuse completed it in the study it was tested in therefore it's efficacy for use among other professionals requires further testing (Conrad et al 2010b).

The following themes emerged in the review as important to tool performance. Recommendations for optimising their use are made.

Length of tool: This aspect is important, as the length of a screening tool, and therefore the length of time to complete it, impacts on whether clinicians (or victims) will use it. Evidence indicates that tools that take longer than an hour to complete are shown to decrease the quality of the data collected and are likely to be met with resistance by those completing them and so are less likely to be used (Fulmer et al, 2004). The Elder Assessment Instrument-Revised at fifty-one items was the longest tool reviewed, followed by the lowa Elder Abuse Screen, the Indicator of Abuse and the Older Adult Financial Exploitation Measure, all with twenty-five items. Although long, the Elder Assessment Instrument-Revised (together with the Elder Assessment Instrument) can be administered in a fifteenminute interview, and allows for a physical examination of the person, provided a clinician is present to undertake the examination. The lowa Elder Abuse Screen, as a combination of three other tools, is 
a more practical length but was only tested in a small sample, making it difficult to fully evaluate efficacy. The Elder Abuse Suspicion Index is brief (it takes two minutes to complete), with six items only, and the doctors that used it in the study found it to be user friendly (Yaffe et al, 2008). The Brief Abuse Screen for the Elderly is also brief but is intended to be used alongside the Indicators of Abuse tool, resulting in a much lengthier assessment time (can be a two to three hour interview) (Reis and Nahmiash,1998: Yaffe et al, 2008). The Hwalek-Sengstock Elder Abuse Tool contains fifteen items but can, in practice, take longer due to some ambiguity of questions. The Vulnerability to Abuse Screening Scale contains twelve items but needs further testing. The Older Adults Psychological Abuse Measure is practicable at eighteen items and has shown to be useful in establishing the severity of abuse. Short screening tools have proved useful in other difficult to detect areas of health and social care, such as intimate partner violence, depression and alcoholism. The ideal tool would be brief but also comprehensive (Yaffe et al, 2008).

Method of administration: The method of administration is central to the process. Without an approach that is sensitive and acceptable, older people are less likely to disclose abuse (or reply accurately). Three methods were found in this review: direct questioning by a professional, self-report and completion by use of computer assisted technology. Direct questioning also allows for a physical observation of the person. Evidence also suggests that most victims will not initiate disclosure but will, when asked directly, admit to experience of abuse (Cohen, 2011). Self-report tools are used effectively in other areas of healthcare dealing with vulnerable populations, (e.g. HIV screening) and may allow for fuller disclosure (Fulmer et al, 2012). Investigation in the field of intimate partner violence has found direct questioning is less preferable when compared with self-completion methods. However there is an increased risk of incomplete responses (MacMillan et al, 2006). Selfcompletion tools also require precise responses from people who may be distressed or suffering some degree of cognitive impairment. Consequently these tools may be less accurate in terms of their application. Computer assisted technology, in which individuals log their responses in a computer programme, was used in the Fulmer 2012 study, has similar issues to self-report but is a new and novel intervention with interesting potential for respondents to use.

Scoring algorithms and cut off points: Some tools use a scoring system or a Likert type scale to report. For example from the Brief Abuse Screen for the Elderly: for the question: 'do you suspect abuse' the response is from $1=$ no, not at all, to $6=$ yes, definitely. The concern with a scoring system is that the weight (or value) an item is given is a more complex process to interpret than simply providing a score. For example, in the Vulnerability to Abuse Screening Scale, the question: 'has anyone close to you tried to hurt you or harm you recently?' could have more serious potential consequences than the question 'can you take your own medication and get around by yourself?' (Fulmer et al, 2008). The Elder Assessment Instrument does not have any scoring algorithm, where other tools, such as the Hwalek-Sengstock Elder Abuse Screening Tool, recommend that if a positive or negative response is given to certain items then suspicion of abuse is evident. An example may be seen in the question: 'do you trust most of the people in your family?', or 'does anyone tell you that you give them too much trouble?' Here a positive response to the first item must be understood in relation to the other items in the tool. This can result in the subjective view of the completing person affecting the tool (Fulmer, 2005). Reliance solely on a screening tool in the health and social care setting is therefore best avoided and ideally their use needs to be a part of a comprehensive system. Screening tools are useful, however, to raise the index of suspicion about abuse, to prompt further investigation and to promote reflection and possible action among professionals.

\section{Conclusion}

Risks and signs:

Inherent in research on screening tools in elder abuse lies the difficulty that, even in the instruments considered by the experts to be the most effective, there remains a lack of distinction between tools screening suspected and actual abuse and tools screening for risk factors (Haggerty et al, 2011). It is important to tease out such nuanced concepts, as the choice of screening tool to use in a clinical setting will be dependent on where on the continuum between signs and risk factors a specific situation fits. Distinguishing between these is essential as the two approaches incorporate differing points of thoroughness and examination (Perel-Levin, 2008). The work of Bass et al gives clarity to these concepts. Figure 2 is adapted from their study and is used to illustrate the process (Bass et al, 2008): 
Figure 2: Flowchart of differences in screening for risk and screening for signs:

Risk of violence/abuse

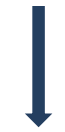

Is there potential abuse/violence

Risk of abuse tool

Actual or suspected abuse/violence

Do you suspect abuse/violence?

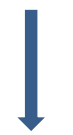

Suspected abuse tool
Is violence reported/observed?

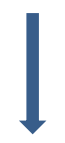

Actual abuse tool

It is concerning to establish a lack of robust evidence for the effectiveness of screening tools to detect elder abuse. This results in an inability to make a recommendation in favour of any particular screening tool. Tools may help with detection of potential abuse, but rather than recommend a 'one size fits all' approach, it is more useful to consider the clinical environment, the individual and the situation rather than propose use of a specific tool for all settings. For example, in a busy Accident and Emergency environment, it is likely that a brief tool, easy to administer and which assesses all types of abuse would be most useful. The EASI tool, although it performed poorly in the diagnostic accuracy testing, is ideally suited to this setting. The Expanded Indicators of Abuse (with good sensitivity) would likely be better suited to a GP Surgery, where more time is generally available, the person may be well known to the Doctor and where the professional may have concerns of a risk of abuse rather than actual signs or symptoms. The Elder Abuse Assessment Instrument, with good specificity, appears best suited to a hospital environment. It allows for a physical examination as part of a comprehensive assessment and is useful where actual signs of abuse are suspected or apparent.

This systematic review has shown little evidence of the rigorous testing of screening tools. As a consequence this makes it important to consider the issue of false positives and false negatives. Consideration of the ethical issues in detecting elder abuse requires careful reflection and attention. Accusing someone of abuse when it is not happening, or missing a case of abuse when it is occurring can have devastating consequences. Is the overall harm of not detecting/leaving someone in a dangerous situation, outweighed by the risk of falsely accusing an individual? Assessment of decisional capacity also needs careful thought and consideration. Screening tools used within an overall system may help to provide a professional with a more objective approach, but this approach must be carefully balanced.

The 2014 Care Act makes it the responsibility of the local authority to make enquires if abuse is reported or suspected. Action on Elder Abuse has commenced a campaign to make elder abuse a punishable crime (Action on Elder Abuse, 2016). A consideration of how professionals currently make decisions around safeguarding in clinical practice, when for example, deliberating on situations such as whether to intervene or not, clearly needs further exploration. It is apparent that many health and social care professionals are currently poorly equipped to manage elder abuse, but they need to know what to do and how to respond if abuse is suspected. Providing them with skills, knowledge and processes is imperative and screening tools are one step in the process to achieve this.

Accurate detection of elder abuse is challenging. Ambiguities in the definitions of elder abuse used have an impact on the performance of screening tools. Throughout the literature review and database search for this review there was no evidence found of either research in the use of screening tools or evidence of their use in clinical practice throughout the UK. Further research on the potential use of screening tools in settings in the UK is required.

\section{Limitations:}


There are a number of limitations to this study and these are a follows. A gold standard tool in elder abuse does not currently exist, which adds to the complexities of their evaluation, as there is no tool to compare against (Yaffe et al, 2008).

Additionally the lack of homogeneity in the included studies made the synthesis of the results challenging. Furthermore, the variations in the characteristics and quality of the tools themselves make applying consistent and comparable analysis of their properties difficult.

It was also not possible, due to resource constraints, to access the paper which reported the original study of the E-IOA. The results of the reported study should be interpreted with caution.

\section{Research recommendations}

Rigorous sensitivity and specificity testing of screening tools is required. A two stage study of a tool, such as the EASI, in a health and social care environment would be beneficial. It would require adaptation to the UK environment followed by a pilot study to refine the tool for use. Then a large scale single blind randomized controlled trial comparing the EASI with a 'treatment as usual' arm could be undertaken to fully test this in practice.

\section{Implications for practice:}

Screening tools are not diagnostic and are used to highlight the need for onward referral or further assessment. Though they require further testing, they can help health and social care professionals to provide a systematic and objective approach to the decision making process.

The overall management of elder abuse requires review and further development. Screening tools in isolation will not be effective. Training, staff supervision and support are required in order to have a system that will handle issues sensitively and effectively.

\section{References:}

Action on Elder Abuse (2017a) Available at: http://elderabuse.org.uk/what-is-elder-abuse/ (Last accessed: 01/07/2017).

Action on Elder Abuse (2017b) Available at https://elderabuse.org.uk/ (Last accessed 01/07/2017)

Bass, D.M., Anetzberger, G.J., Ejaz, F.K. and Nagpaul, K. (2008) 'Screening Tools and Referral Protocol for Stopping Abuse among Older Ohioans: A Guide for Service Providers', Journal of Elder Abuse and Neglect, 13(2), pp. 23-28. Biggs S, Erens B, Doyle M et al (2013) Abuse and Neglect of Older people: Secondary analysis of UK prevalence study. National Centre for Social Research: Available at: http://www.natcen.ac.uk/media/20824/abuse-neglect-older-people.pdf

(Last accessed: 31/07/2016).

Bitondo Dyer, C., Pavlik, V., Murphy, K. et al (2000) 'The High Prevalence of Depression and Dementia in Elder Abuse or Neglect'. Journal of American Geriatrics Society, 48, 2, 205-208

Buri, H., Daly, J., Gerald, J. (2009) Elder Abuse Telephone Screen Reliability and Validity. Journal of Elder Abuse and Neglect. 21, 1, 58-73

Cohen, M. (2013) The Process of Validation of a Three-Dimensional Model for the Identification of Abuse in Older Adults, Archives of Gerontology and Geriatrics, 57, 3, 243-249.

Cohen, M. (2011) 'Screening Tools for the Identification of Elder Abuse', Journal of Clinical Outcomes Management, 18, 6, 261-270

Conrad, K., Iris, M. and Ridings, J. (2010a) Self-Report Measure of Financial Exploitation of Older Adults, The Gerontologist. 50, 6, 758-773.

Conrad, K., Iris, M, and Ridings, J. (2010b) Self-Report Measure of Psychological Abuse of Older Adults, The Gerontologist. 51, 3, 354-366.

Cooper, C., Selwood, A. and Livingston, G. (2008) The Prevalence of Elder Abuse and Neglect: A Systematic Review, Age and Ageing. 37, 151-160. 
Darzins, P., Lowndes, G., Wainer, J., Owada, K. and Mihaljcic, T. (2009) Financial Abuse of Elders: A Review of the Evidence. Protecting Elder's Assets Study. Melbourne: Monash Institute of Health Research, Nursing and Health Services.

Fulmer, T., Guadagno, L., Bitondo Dyer, C. et al (2004) 'Progress in Elder Abuse Screening and Assessment Instrument', Journal of American Geriatrics Society, 52, 297-304

Fulmer, T., Paveza, G., VandeWeerd, C. et al (2005) Neglect Assessment in Urban Emergency Departments and Confirmation by an Expert Clinical Team. Journal of Gerontology. 60A, 8, $1002-$ 1006

Fulmer T (2008) 'Screening for Mistreatment of Older Adults', American Journal of Nursing. 108, 12, $52-59$

Fulmer, T., Strauss, S., Russell, S. et al (2012) Screening for elder mistreatment in dental and medical clinics. Gerontology. 29, 96-105.

Grimes, D.A. and Schulz, K.F. (2002) 'Uses and Abuses of Screening Tests', The Lancet, 359, 881884

Haggerty, L.A., Hawkins, J.W. and Fontenot-O' Connor, A. (2011) Tools for Screening Interpersonal Violence: State of the Science, Violence and Victims, 26, 6, 725-737.

Lachs, M.S., Williams, C.S., O'Brien, S., Pillemer, K.A. and Charlson, M.E. (1998) The Mortality of Elder Mistreatment, Journal of American Medical Association. 280, 5, 428-432.

Lindenbach, J.M., Larocque, S., Lavoie, A.M. and Garceau, M.L. (2011) Older Adult Mistreatment Risk Screening: Contribution to the Validation of a Screening Tool in a Domestic Setting, Canadian Journal on Ageing, 31, 2, 235-252.MacMillan, H.L., Wathen, C.N., Jamieson, E., Boyle, M., McNutt, L.A., Worster, A., Lent, B. and Webb, M. (2006) Approaches to Screening for Intimate Partner Violence in Health Care Settings, Journal of the American Medical Association, 296, 6, 530-536.

Moher, D., Liberati, A., Tetzlaff, J. et al (2009) Preferred Reporting Items for Systematic Reviews and Meta-Analyses: The PRISMA Statement, British Medical Journal. 305, 998- 999.

Moody, L., Voss, A. and Lengacher, C. (2000) Assessing Abuse Among the Elderly Living in Public Housing. Journal of Nurse Management. 8, 1, 61-70.

National Research Council (2003) Elder Mistreatment: Abuse, Neglect, and Exploitation in an Aging America. Panel to Review Risk and Prevalence of Elder Abuse and Neglect. Washington DC, The National Academies Press.

Naughton, C., Drennan, J. and Lyons, I. (2012) Elder abuse and Neglect in Ireland: Results from a National Prevalence Survey, Age and Ageing. 41, 98-103.

O'Keeffe, M., Hills, A., Doyle, M. et al (2007) UK Study of Abuse and Neglect of Older People: Prevalence Survey Report. London: National Centre for Social Research.

Penhale, B. (1993) The Abuse of Elderly People: Considerations for Practice. British Journal of Social Work. 23, 2, 95-112

Phelan, A. and, Treacy, M. (2011) A Review of Elder Abuse Screening Tools for use in the Irish Context. National Centre for the Protection of Older People: University College Dublin.

Phelan, A., Fealy, G. and Downes, C. (2014) The Older Adult Financial Exploitation Measure: A Pilot Study to Test its Appropriateness in an Irish Context. National Centre for the Protection of Older People: University College Dublin.

Popay, J., Roberts, H. and Sowden, A. (2006) Guidance on the Conduct of Narrative Synthesis in Systematic Reviews, Version 1. Available at: http://www.lancaster.ac.uk/shm/research (Last accessed: 01/04/2015: with permission from the University). 
Pritchard, J. (1995) The Abuse of Older People, A Training Manual for Detection and Prevention. $2^{\text {nd }}$ edition. Wiltshire: Jessica Kingsley Publications

Raffle, A. and Gray, M. (2007) Screening Evidence and Practice. Oxford: Oxford University Press

Reis, M. and Nahmiash, D. (1998) Validation of the Indicators of Abuse Screen. The Gerontological Society of America. 38, 4, 471-480.

Ryan, R; Cochrane Consumers and Communication Review Group (2013) Cochrane Consumers and Communication Review Group: Data Synthesis and Analysis. http://cccrg.cochrane.org (last accessed: 13/06/2016.

Schofield, M. and Mishra, G. (2003) Validity of Self-Report Screening Scale for Elder Abuse: Women's Health Australia Study, The Gerontologist. 43, 1, 110-120.

Yaffe, M., Wolfson, C., Lithwick, M. et al (2008) Development and Validation of a Screening Tool to Improve Physician Identification of Elder Abuse, Journal of Elder Abuse and Neglect. 20, 3, 276-300.

Yaffe, M., Weiss, D. and Lithwick, M. (2012) Seniors Self-Administration of the Elder Abuse Suspicion Index (EASI): A Feasibility Study, Journal of Elder Abuse and Neglect. 24, 4, 277-292

World Health Organisation (2008) A Global Response to Elder Abuse and Neglect. Available at: http://www.who.int/ageing/publications/ELDER DocAugust08.pdf (last accessed 01/07/2017). 